\title{
PEAK-TO-AVERAGE POWER RATIO REDUCTION IN AN FDM BROADCAST SYSTEM
}

\author{
Zhengya Zhang, Renaldi Winoto, Ahmad Bahai, and Borivoje Nikolić \\ Department of Electrical Engineering and Computer Sciences \\ University of California, Berkeley
}

\begin{abstract}
Digital predistortion is a technique used to reduce the signal dynamic range in a multichannel system in order to improve power amplifier (PA) efficiency. These techniques have been developed for orthogonal frequency-division multiplexing (OFDM) systems, but are not directly applicable to general frequency-division multiplexing (FDM) systems such as those used in broadcast. A modification to the tone injection approach allows constellation translations to be directly applied at the digital intermediate frequency (IF) domain and provides redundancy in lowering peak-toaverage ratio (PAR). Simulation of a 24-channel 256-QAM FDM cable television transmission system shows a $3.3 \mathrm{~dB}$ PAR reduction at $10^{-6}$ clipping probability.
\end{abstract}

Index Terms - multichannel systems, power amplifiers, peak-to-average ratio, digital predistortion, tone injection.

\section{INTRODUCTION}

A major drawback of a multichannel system is the relatively high amplitude envelope variation that is inherent to the transmitted signal [1]. The envelope variation is often quantified using the peak-to-average ratio (PAR). Large signal peaks occur in the transmitted signal when the signals in many of the subchannels add constructively in phase. Such large signal peaks may result in clipping of the signal voltage in a digital-to-analog converter (DAC) and it may saturate the power amplifier (PA), causing intermodulation distortion in the transmitted signal. As more subchannels are integrated in the system, envelope variation increases and distortion becomes more severe.

Large signal dynamic range can be reduced in orthogonal frequency-division multiplexing (OFDM) systems by digitally predistorting the transmitted signal before it enters the DAC. Predistortion compresses the signal dynamic range and reduces the PA's operating range, thus linearizes the PA without significant power back-off. Advances in digital communication techniques and proliferation of new interactive services are driving the migration of broadcast systems towards using digital IF. For this to be successful the PAR problem needs to be addressed. This work analyzes existing digital predistortion techniques and proposes a PAR-reduction solution that can be applied to a general frequency-division multiplexing (FDM) system.

Various methods have been formulated to achieve PAR reduction in multicarrier OFDM systems. The simplest methods are based on the observation that a high signal peak is created when signals in multiple channels add constructively in phase. Such methods attempt to systematically insert phase shifts to subcarriers to reduce their in-phase probability. Various algorithms have been proposed in determining the optimal phase selections [2-5]. Coding is another promising approach that systematically adds redundancy to avoid certain symbol combinations that cause high signal peaks [6-11]. Coding also enhances error tolerance of the system. Recent efforts explore the possibility of varying signal constellation in order to mitigate high PAR. A controlled amount of constellation error can be introduced to gain an appreciable amount of PAR reduction [12]. Methods that expand outer constellation points effectively reduce PAR without sacrificing error performance $[13,14]$. A method known as tone injection projects the entire constellation to an expanded domain to improve flexibility in PAR reduction [15].

In an OFDM system, isolation between subchannels is guaranteed by choosing the subchannel spacing equal to the symbol rate. In contrast, a general FDM system achieves isolation between subchannels through filtering. Filtering produces correlations between input symbols, which make it difficult for an algorithm to associate any modification of the baseband symbol to the resulting change in the PAR of the filtered and upconverted signal. This difference is critical with respect to choosing an appropriate PAR reduction scheme, as the correlation between input symbols renders most established PAR reduction schemes for OFDM systems impractical in this general FDM system.

We propose a modified tone-injection approach suitable for FDM systems. By injecting tone(s) and evaluating the resulting PAR reduction directly in the digital IF domain, no knowledge of the peak-causing baseband symbols or symbol combinations is needed. This modification makes the algorithm amenable for an efficient implementation. In this paper, we apply the proposed technique to a multichannel digital cable television transmission system specified by the data-over-cable service interface specification (DOCSIS) standard [16]. 


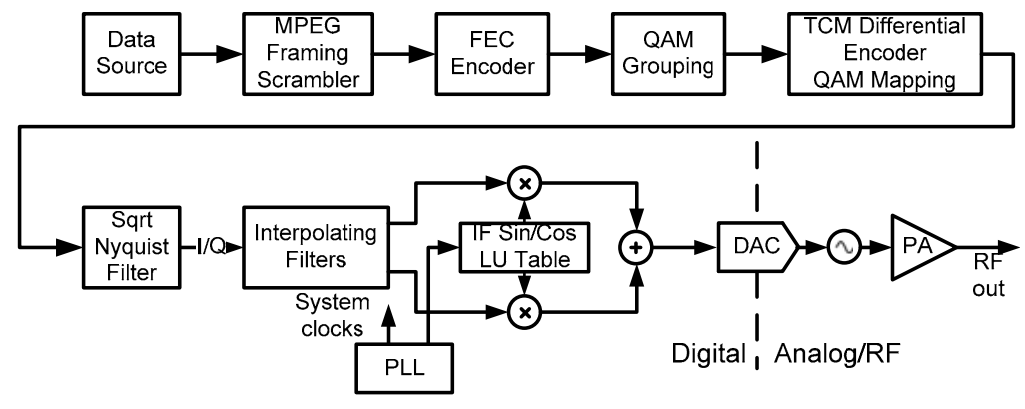

Figure 1. QAM Modulator.

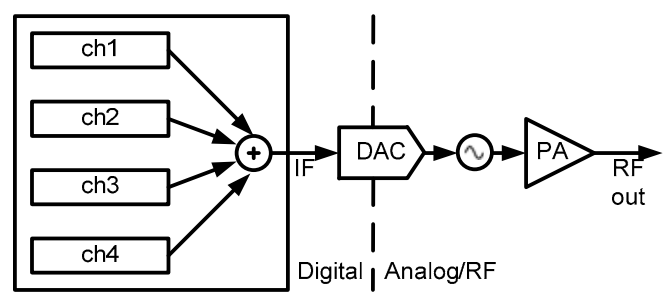

Figure 2. Multichannel integration.

\section{SYSTEM DESCRIPTION}

In current digital cable television transmission system, each channel is implemented as a stand-alone quadrature amplitude modulation (QAM) module from digital baseband to RF output (Fig 1) [16]. A combination of digital, analog, and RF components are used to generate each channel. A number of these QAM modules can be placed into a single system card. The outputs of the QAM modules are passively combined at RF to feed a single coax line. The lack of any component sharing between channels in this scheme results in a high system cost. Furthermore, passive signal combining at the high-power RF output can be highly power inefficient because signal loss at this stage results in a significant power loss for the system as a whole.

Advances in digital VLSI and data converter technologies have made it possible to generate and combine several QAM channels digitally at an intermediate frequency. In this multichannel implementation, several QAM channels can share a single chain of analog and RF blocks. An illustration of this approach is shown in Fig. 2.

As with any multicarrier system, adding multiple channels together results in large envelope variations. Consequently, better analog and RF parts are required to cope with a much larger signal dynamic range. An alternative approach is to reduce the signal dynamic range to relax the requirement on the analog and RF parts. If the approach is applied in the digital domain, it is called digital predistortion.

\subsection{System Constraints and Prototype Design}

The system under investigation is a multichannel FDM system. The DOCSIS standard requires the spectral mask to be complied on each subchannel. Each subchannel has to be carefully filtered in the baseband to avoid corruption of neighboring frequency bands. The standard does not provide any spare bandwidth. The assumption is that all subchannels are in use for data transmission.
Due to the broadcast nature of the system, PAR reduction should require minimal change to the receiver. The overhead with the PAR reduction solution should be minimized and it should not affect transmission spectral shape or bit error rate (BER) performance.

A multichannel prototype system based on DOCSIS 2.0 specifications [16] is built to investigate digital predistortion techniques. The system uses a 256-QAM modulation scheme. Subchannels are spaced $6 \mathrm{MHz}$ apart and the bandwidth of each subchannel is $5.36 \mathrm{MHz}$. Frequency response is root-raised cosine shaped with a roll-off factor of 0.12 . The transmitter is divided into IF and RF stages. The first stage modulates baseband signal to IF. Multiple subchannels are digitally combined at the end of the IF stage and passed to the RF modulation stage in the analog domain. The PAR investigation is performed on the combined IF signal. The structure of the system follows the block diagram demonstrated in Fig. 2. RF and analog components are simulated by a digital upconverter. Framing and channel encoding are omitted, as they do not influence the PAR investigation.

The transmission spectral mask specifies an out-ofband rejection of $-58 \mathrm{dBc}$ at RF. Sufficient margins should be allocated for analog and RF impairments. The Nyquist filter employs a minimum 200-tap FIR with 14 bits of word length to meet the spectral-mask requirement. The interpolation filter is implemented using two 6-stage cascaded integrator-comb (CIC) filters. The total latency is over 200 clock cycles from data source to IF output.

\section{PAR REDUCTION BY MODIFIED TONE INJECTION}

PAR reduction by transmitting side information or coding is disallowed in the television transmission systems, because there is no spare bandwidth available. Constellation modification is the only suitable approach, and will be analyzed in this section. 


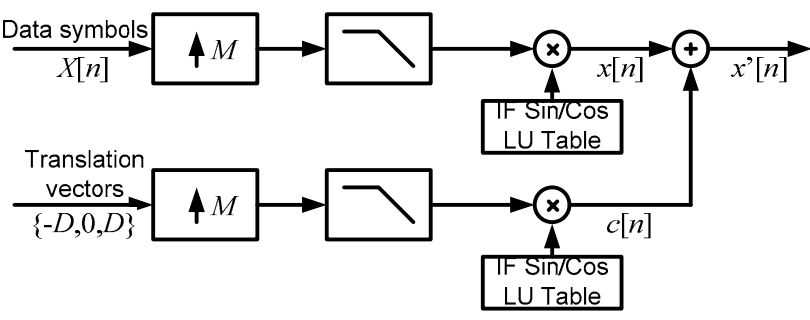

Figure 3. FDM multichannel system with tone injection at IF.

\subsection{Constellation Modification}

One solution in constellation modification is to push the error vector magnitude (EVM) envelope. Good results have been shown [12] for up to 64-QAM using 52 data carriers and 12 free carriers. A system without any free carriers would not realize much of the PAR reduction. Furthermore, a large constellation, such as a 256-QAM system in consideration, requires a smaller EVM. This, in turn, reduces the margin for PAR reduction. An alternative scheme called active constellation extension (ACE) [14] moves outer constellation points. Outer constellation points are not as tightly constrained, but the proportion of outer constellation points decreases with larger constellations, which limits the effectiveness of the ACE scheme.

Tone injection [15] allows every constellation point to move; however the move is restricted to fixed length and directions. This constraint reduces the search space and hence the implementation cost. A minor drawback with the tone injection technique is the addition of a modulo operation to the receiver. This addition can be easily absorbed in the digital domain, although it might not be applicable to some legacy receivers.

There are two general schemes of implementing constellation modification: feedback or feed-forward. Feedback approaches are direct mappings of recursive optimization algorithms; feed-forward approaches are often based on simplified approximations. In a feedback system, IF signals are optimized for lower peaks by varying the baseband signals in a loop that encloses both the modulator and the demodulator. A feedback approach is suitable for OFDM systems, where efficient IFFT and FFT algorithms are used for modulation and demodulation. In comparison, the FDM system under investigation is not amenable to a feedback implementation, because both modulator and demodulator contain complex baseband filtering stages whose latency can be in excess of hundreds of clock cycles. If such a modulator and a demodulator are to be enclosed in a feedback loop, the long loop delay makes a stable and effective feedback implementation difficult if not impossible.

In the following sections, we describe a feed-forward implementation of the tone injection algorithm.

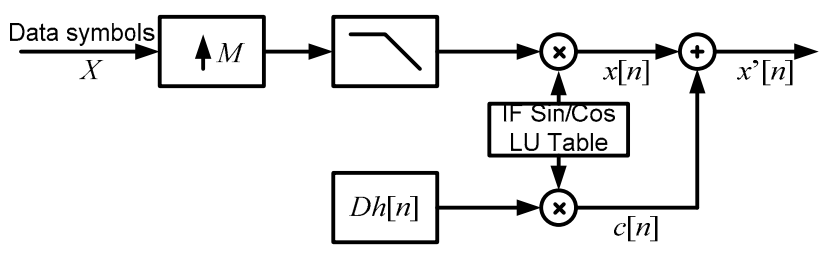

Figure 4. FDM multichannel system with pre-upsampled and filtered translation vector injected at IF.

\subsection{Translation of Constellation Points}

Tone injection projects constellation points to an extended domain [15], which can be interpreted as adding a translation vector to the baseband symbol. Baseband filtering in each subchannel creates a correlation window between symbols. Therefore, a peak identified in the combined IF data stream does not directly lead to the identification of the particular baseband symbols that cause the peak. The solution is to perform constellation extension at IF instead of baseband, because it eliminates the need to identify the peakcausing baseband symbols, such that the PAR-reduction algorithm can be efficiently implemented in a feed-forward manner.

Performing constellation extension at IF requires that the translation vector to pass through upsampling, filtering, and IF modulation to keep the transmission spectrum clean, a block diagram of which is shown in Fig. 3. The translation vector moves the in-phase and/or quadrature components of the constellation point by a distance $D$, therefore the upsampled and filtered translation vector is the filter impulse response $h[n]$ scaled by $D$. The sequence $D h[n]$ can be stored in a buffer. The system block diagram is simplified and illustrated in Fig. 4.

An $L$-tap FIR filter in the baseband implementation creates a correlation window of size $L$. Translation of one baseband symbol influences the entire correlation window. Consequently, the translation vector $D h[n]$ needs to cover the entire correlation window and the IF data sequence $x[n]$ (see Fig. 4 for notation) is also framed in a window of size $L$ to facilitate additions and subtractions. To properly align sequences for additions and subtractions, we divide $x[n]$ into subframes of size $M$, which equals to the upsampling rate. The upconverted translation vector, $c[n]$, is conveniently aligned with $x[n]$ at its subframe boundaries.

\subsection{Modified Tone Injection Algorithm Description}

The objective of tone injection is to add or subtract translation vector $D h[n]$ at selected subchannel(s) to mitigate peaks in the IF data sequence $x[n]$. In single-tone injection, the translation vector $D h[n]$ is applied to only one of the subchannels to get the most peak reduction. 
The channel and the direction to which the translation vector is applied must somehow be determined. A simple algorithm can be formulated to compare the possible choices and select one that provides the lowest PAR. Suppose the correlation window length is $L$, baseband symbol time is $T$, upsampling rate is $M$, and the number of subchannels is $N$. The algorithm is described through the following steps.

0 . Frame a window of $L$ samples in the IF data sequence. The window starts with $x\left[n_{0}\right]$ and ends with $x\left[n_{L-1}\right]$. Both $n_{0}$ and $n_{L-1}$ are required to be positioned at subframe boundaries.

1. For each subchannel $k$ with IF frequency $\omega_{k}$, calculate the IF translation vector:

$$
\begin{aligned}
& c_{k}[i]=D h[i]\left\{ \pm d_{1} \cos \left(\frac{\omega_{k} n_{i} T}{M}\right) \pm d_{2} \sin \left(\frac{\omega_{k} n_{i} T}{M}\right)\right\} \\
& 0 \leq i \leq L-1,0 \leq k \leq N-1, \text { and } d_{1}, d_{2} \in\{0,1\} .
\end{aligned}
$$

2. Apply IF translation vector to IF data sequence to obtain candidate solutions:

$r_{k}[i]=x\left[n_{i}\right]+c_{k}[i], 0 \leq i \leq L-1,0 \leq k \leq N-1$.

3. Select one of the sequences among $\left\{r_{k}, 0 \leq k \leq N-1\right\}$ that gives the lowest PAR. Let the sequence be $r_{\text {opt }}$.

- If the PAR of $r_{\text {opt }}$ is lower than that of $x[n], n_{0} \leq n \leq n_{L-1}$, replace $x[n], n_{0} \leq n \leq n_{L-1}$ with $r_{\text {opt }}[i], 0 \leq i \leq L-1$.

- If the PAR of $r_{o p t}$ is higher than that of $x[n], n_{0} \leq n \leq n_{L-}$ $1, x[n]$ remains unchanged.

4. Shift out $M$ samples from the IF data frame, $x\left[n_{0}\right], x\left[n_{1}\right]$, $x\left[n_{2}\right], \ldots, x\left[n_{M-1}\right]$. Shift in $M$ new samples to the frame. Restart the algorithm from Step 1. If encounter end of $x[n]$, stop.

\subsection{Implementation}

We built a multichannel 256-QAM DOCSIS 2.0 digital transmitter using a Xilinx Virtex II Pro (XC2VP70) FPGA. 4 transmit channels are integrated in one FPGA. The IF sampling frequency is set to $160 \mathrm{MHz}$. A summary of the available resources and the area utilization of the system are listed in Table I.

TABLE I

AREA Utilization OF A XILINX XC2VP70 FPGA FOR A 4CHANNEL 256-QAM TX SYSTEM.

\begin{tabular}{|c|c|c|c|c|}
\hline & $\begin{array}{c}\text { Avail- } \\
\text { able } \\
\text { resources }\end{array}$ & $\begin{array}{c}\text { Base } \\
\text { system }\end{array}$ & $\begin{array}{c}\text { Single- } \\
\text { tone } \\
\text { injection }\end{array}$ & $\begin{array}{c}\text { Single- } \\
\text { tone two- } \\
\text { iteration }\end{array}$ \\
\hline Slice flip flops & 66,176 & 54,009 & 58,119 & 61,967 \\
\hline 4 input LUTs & 66,176 & 42,331 & 46,803 & 51,147 \\
\hline Block RAMs & 328 & 42 & 46 & 46 \\
\hline Multipliers & 328 & 8 & 16 & 16 \\
\hline
\end{tabular}

A single-tone injection applied to this 4-channel transmitter provides 4 choices of tones and 8 directions to translate constellation for a total of $4 \times 8=32$ selections to modify the IF data stream. The optimal low-peak sequence among the 32 choices can be selected by a multiplexer tree. Implementation costs associated with tone injection consist of the multiplications to compute translation vectors, the additions to find candidate sequences, the buffering and the peak detection of the sequences, and a multiplexer tree to select the optimal sequence. The costs of multipliers, adders, buffers, peak detectors, and the multiplexer tree are proportional to the number of transmit channels, thus the area complexity is $O(N)$, where $N$ denotes the number of subchannels.

We designed a single-tone injection system for the 4channel 256-QAM transmitter. The area utilization of the system is listed in Table I. The numbers of slice flip flops, 4 input LUTs, and block RAMs increase by approximately $10 \%$ compared to the base system. The number of multipliers doubles. In this specific FPGA implementation, dedicated multipliers are allocated without additional area overhead.

Two-tone and multi-tone injections incur much higher implementation costs though they offer potentially higher PAR reductions. A two-tone injection applied to the 4channel system provides $C(4,2)=6$ choices of tones and 8 $\times 8=64$ directions to translate constellation for a total of 6 $\times 64=384$ selections to modify the IF data stream. In general, the area complexity of a multi-tone injection system is $O\left((8 N)^{t}\right)$, where $t$ denotes the number of tones to be injected and it is assumed to be a small number. Multi-tone injection can be approximated by repeated single-tone injections. The repetition can be unrolled to achieve a higher throughput. The area complexity of the unrolled iterative tone injection is $O(r \cdot N)$, where $r$ denotes the number of iterations. The area utilization of a single-tone two-iteration injection system is listed Table I for comparison. The incremental resource utilization is less than $10 \%$.

\section{RESULTS}

A complementary cumulative distribution function (CCDF) plot can be used to quantify the PAR of a signal as a function of the clipping probability. The CCDF plots in Fig. 5 demonstrate the increase of PAR when more channels are combined. A 24-channel system has a PAR of $13.8 \mathrm{~dB}$ at $10^{-6}$ clipping probability, which is $2.8 \mathrm{~dB}$ higher than a single-channel system.

The PAR-reduced signals using single-tone injection are characterized by the CCDF plot in Fig. 6. At $10^{-6}$ clipping probability, the PAR is reduced from $13.8 \mathrm{~dB}$ to 12.2 $\mathrm{dB}$, a saving of $1.6 \mathrm{~dB}$. Power spectral density (PSD) of the PAR-reduced signal is shown in Fig. 7. 


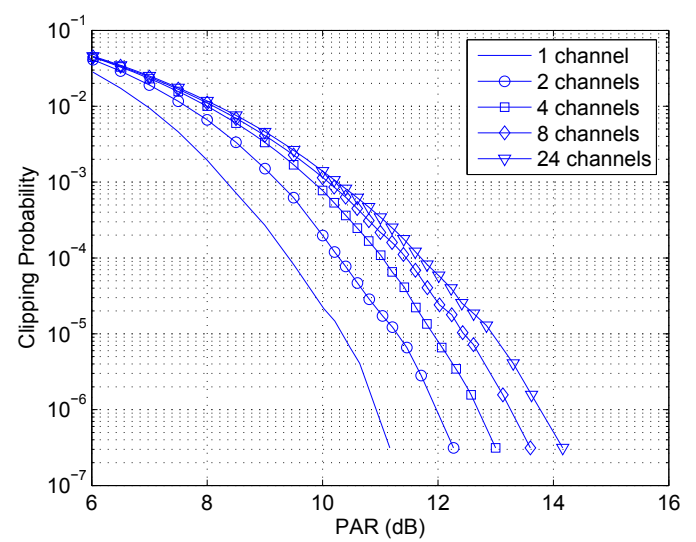

Figure 5. Adding subchannels causes an increase in PAR.

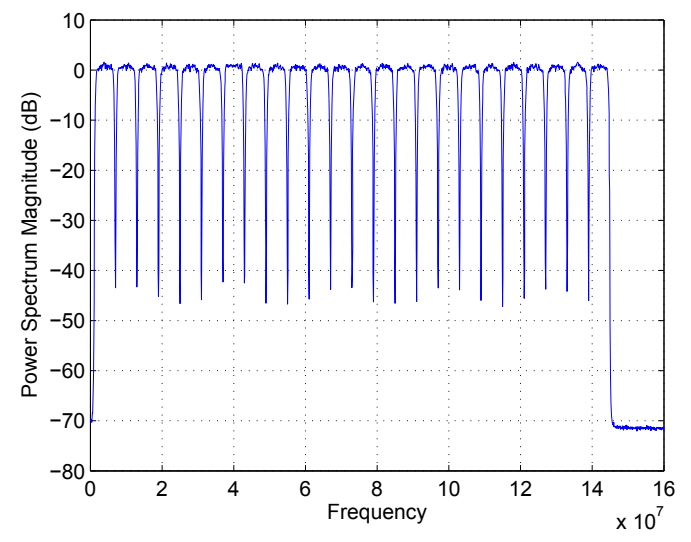

Figure 7. Power spectral density of the PAR-reduced signal.

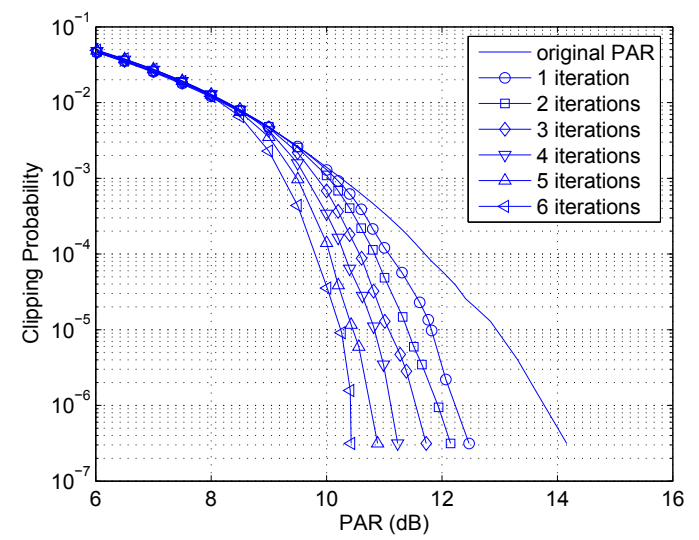

Figure 9. PAR reduction with more iterations.

The tone injection approach can be compared with clipping, which is frequently used in practical systems. To match the $1.6 \mathrm{~dB}$ of PAR reduction by single-tone injection, clipping can be set at a level of $3.94 \times 10^{-5}$ which means that the top $3.94 \times 10^{-5}$ of the peaks are clipped. The resulting CCDF plot is shown in Fig. 6. PSD of the PAR-reduced

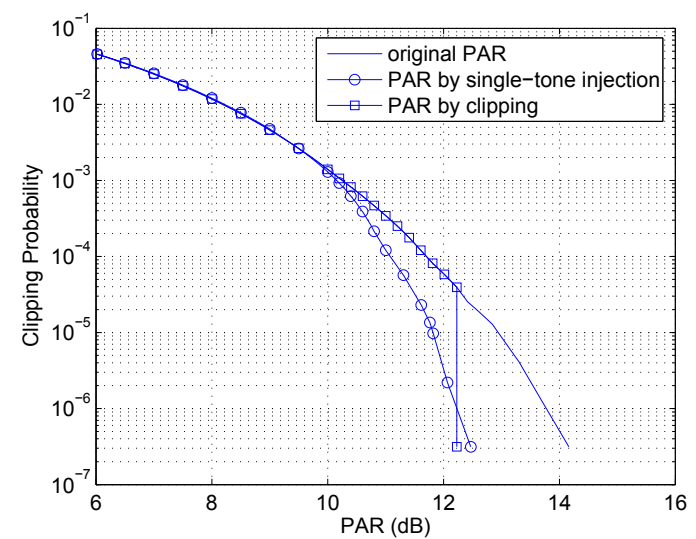

Figure 6. PAR reduction with single-tone injection compared to clipping.

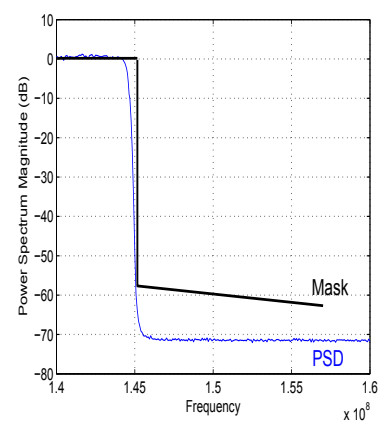

(a)

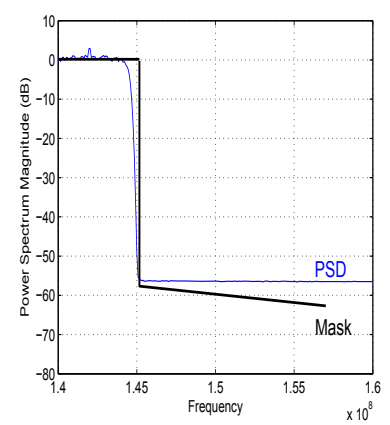

(b)
Figure 8. Enlarged view of (a) the PSD of PAR-reduced signals obtained by tone injection, and (b) the PSD of PAR-reduced signals obtained by clipping.

signals obtained by tone injection and clipping are illustrated side-by-side in Fig. 8. The spectral mask is superimposed on both PSD plots. Unlike tone injection, clipping causes severe spectral regrowth which could violate the spectral mask.

A larger PAR reduction can be achieved by modifying the IF data stream iteratively. Fig. 9 shows the CCDF of the signal amplitudes after more iterations using single-tone injection. At $10^{-6}$ clipping probability, the first iteration reduces the PAR by $1.6 \mathrm{~dB}$. Each additional iteration reduces it further by about $0.3 \mathrm{~dB}$. After 6 iterations, a $3.3 \mathrm{~dB}$ of PAR reduction is achieved.

\section{CONCLUSIONS}

Under the constraints of the FDM system, a survey of OFDM PAR-reduction techniques yields the possibility of adapting the tone injection approach to solve the PARreduction problem in the FDM system. A multichannel 256-QAM prototype for digital cable television transmission is set up to characterize PAR and used as a test platform for PAR reduction. 
In an FDM system, the same OFDM tone injection algorithm cannot be applied due to the difficulty in locating peak-causing baseband symbols and the latency of the feedback loop. The problem can be solved in the digital IF domain using a modified tone injection approach. The modified approach explores the choices in directly adjusting IF data stream using upsampled and filtered translation vectors. A feed-forward algorithm is proposed to select a low-PAR sequence among all the choices. Choices available in modification of the IF stream eliminates the need to pinpoint exactly which symbols in the baseband cause peaks in IF. It is adequate to identify one of the choices in modifying the IF stream to achieve the lowest PAR.

Modified tone injection can be extended by performing more iterations or multi-tone injection to reduce PAR even further. The modified tone injection approach is an effective means of reducing PAR in an FDM system. In the 24channel 256-QAM system, it provides $3.3 \mathrm{~dB}$ of PAR reduction (at $10^{-6}$ clipping probability) with 6 -iteration singletone injection. The transmit spectrum satisfies the spectral mask and BER performance is guaranteed by the underlying tone injection method.

\section{ACKNOWLEDGEMENT}

The authors wish to acknowledge the contributions of the students, faculty, and sponsors of Berkeley Wireless Research Center and the National Science Foundation Infrastructure Grant No. 0403427. The authors would like to thank Chuck Naegeli for discussions and help in defining system requirements, as well as Cisco Systems for supporting this research.

\section{REFERENCES}

[1] A.R.S. Bahai, B.R. Saltzberg, M. Ergen, Multi-carrier Digital Communications: Theory and Applications of OFDM, Springer, 2004.

[2] S.H. Muller, J.B. Huber, "OFDM with reduced peak-to-average power ratio by optimum combination of partial transmit sequences," IEE Electronics Letters, vol. 33, iss. 5, Feb. 1997, pp. 368-369.
[3] R. Bauml, R. Fischer, J. Huber, "Reducing the peak-to-average power ratio of multicarrier modulation by selected mapping," IEE Electronics Letters, vol. 32, iss. 22, Oct. 1996, pp. 2056-2057.

[4] L.J. Cimini Jr., N.R. Sollenberger, "Peak-to-average power ratio reduction of an OFDM signal using partial transmit sequences," IEEE Communications Letters, vol. 4, iss. 3, Mar. 2000, pp. 86-88.

[5] C. Tellambura, "Phase optimisation criterion for reducing peak-to-average power ratio in OFDM," IEE Electronics Letters, vol. 34, iss. 2, Jan. 1998, pp. 169-170.

[6] A.E. Jones, T.A. Wilkinson, S.K. Barton, "Block coding scheme for reduction of peak to mean envelope power ratio of multicarrier transmission schemes," IEE Electronics Letters, vol. 30, iss. 25, Dec. 1994, pp.2098-2099.

[7] T.A. Wilkinson, A.E. Jones, "Minimisation of the peak to mean envelope power ratio of multicarrier transmission schemes by block coding," IEEE Vehicular Technology Conference, vol. 2, Jul. 1995, pp. 825-829.

[8] B.M. Popovic, "Synthesis of power efficient multitone signals with flat amplitude spectrum," IEEE Transactions on Communications, vol. 39, Jul. 1991, pp. 1031-1033.

[9] R.D.J. van Nee, "OFDM codes for peak-to-average power reduction and error correction," IEEE Global Telecommunications Conference, vol. 1, Nov. 1996, pp. 740-744.

[10] J.A. Davis and J. Jedwab, "Peak-to-mean power control in OFDM, Golay complementary sequences and Reed-Muller codes," IEEE Transactions on Information Theorey, vol. 45, Nov. 1997, pp. 2397-2417.

[11] K.G. Paterson, "Generalized Reed-Muller codes and power control in OFDM modulation," IEEE Transactions on Information Theory, vol. 46, Jan. 2000, pp. 104-120.

[12] A. Aggarwal, T.H. Meng, "Minimizing the peak-to-average power ratio of OFDM signals via convex optimization," IEEE Global Telecommunications Conference, vol. 4, Dec. 2003, pp. 2385- 2389.

[13] Y. Kou, W. Lu, A. Antoniou, "New peak-to-average powerratio reduction algorithms for multicarrier communications," IEEE Transactions on Circuits and Systems I: Regular Papers, vol. 51, iss. 9, Sep. 2004, pp. 1790- 1800.

[14] B.S. Krongold, D.L. Jones, "PAR reduction in OFDM via active constellation extension," IEEE Transactions on Broadcasting, vol. 49, iss. 3, Sep. 2003, pp. 258- 268.

[15] J. Tellado-Mourelo, Peak to average power reduction for multicarrier modulation, Doctoral dissertation, Dept. of Electrical Engineering, Stanford University, Stanford, 1999.

[16] DOCSIS 2.0, Data-over-cable Service Interface Specifications, Cable Television Laboratories, Dec. 2001. 